\title{
Verification of Antiparallel G-Quadruplex Structure in Human Telomeres by Using Two-Photon Excitation Fluorescence Lifetime Imaging Microscopy of the 3,6-Bis(1-methyl-4-vinylpyridinium)carbazole Diiodide Molecule
}

\author{
Cheng-Chung Chang, ${ }^{\dagger}$ Jen-Fei Chu, ${ }^{\dagger, \ddagger}$ Fu-Jen Kao,, ,,$\|$ Yi-Chun Chiu,, Pei-Jen Lou, ${ }^{\perp}$ Huei-Chin Chen, ${ }^{\perp}$ and \\ Ta-Chau Chang*,t,§
}

Institute of Atomic and Molecular Sciences, Academia Sinica, P. O. Box 23-166, Taipei, 106, Taiwan, Republic of China, Department of Chemistry, National Taiwan Normal University, Taipei, Taiwan, Republic of China, Institute of Biophotonics Engineering, National Yang-Ming University, Taipei, 11221, Taiwan, Republic of China, Institute of Electro-Optical

Engineering, National Yat-sen University, Kaohsiung, 80424, Taiwan, Republic of China, and Department of Otolaryngology, National Taiwan University Hospital, Taipei, Taiwan, Republic of China

Different G-quadruplex structures for the human telomeric sequence $d\left(T_{2} A G_{3}\right)_{4}$ in vitro have been documented in the presence of sodium and potassium. Verification of the G-quadruplex structures in human telomeres in vivo is the main issue in establishing the biological function of the G-quadruplex structures in telomeres as well as the development of anticancer agents. Here we have applied two-photon excitation fluorescence lifetime imaging microscopy to measure the fluorescence lifetime of the BMVC molecule upon interaction with various DNA structures. The distinction in lifetime measured with submicrometer spatial resolution in two-photon excitation fluorescence lifetime imaging microscopy provides a powerful approach not only to verify the existence of the antiparallel G-quadruplex structure in human telomeres but also to map its localizations in metaphase chromosomes.

Telomeres, the ends of chromosomes, are essential for genome integrity and chromosome replication. ${ }^{1,2}$ Telomeres normally contain tandem repeats of guanine-rich (G-rich) motifs, for example, the hexameric repeats of TTAGGG/CCCTAA in vertebrate telomeres. Of special interest is that the 3 '-overhang G-rich single strand with 50-200 bases could adopt G-quadruplex structures under physiological conditions. Since the folding of telomeric DNA into a G-quadruplex structure has been shown to

\footnotetext{
* To whom correspondence should be addressed. E-mail: tcchang@ po.iams.sinica.edu.tw.

$\dagger$ Academia Sinica.

₹ National Taiwan Normal University.

§ National Yang-Ming University.

" National Yat-sen University.

${ }^{\perp}$ National Taiwan University Hospital.

(1) Blackburn, E. H.; Greider, C. W. Telomeres; Cold Spring Harbor Laboratory Press: New York, 1996.

(2) Williamson, J. R. Annu. Rev. Biophys. Biomol. Struct. 1994, 23, 703-730.
}

inhibit telomerase activity in vitro, molecules that stabilize Gquadruplex structures have the potential to interfere with telomere replication and possibly to serve as anticancer agents. ${ }^{3-5}$ Although direct evidence for the presence of G-quadruplex structures in vivo has been reported in the cilitate Stylonychia, ${ }^{6}$ the promoter of c-myc, ${ }^{7}$ and the human telomeres, ${ }^{8}$ the existence of Gquadruplex structures in human cells is still in debate. ${ }^{9}$

To distinguish the very small amounts of G-quadruplex structures from the overwhelming amounts of duplex structures in chromosomes, a 3,6-bis(1-methyl-4- vinylpyridinium) carbazole diiodide (BMVC, U.S. patent 2005-0090671) molecule was synthesized to recognize the unimolecular G-quadruplex structure of human telomeric sequence of $d\left(\mathrm{~T}_{2} \mathrm{AG}_{3}\right)_{4}(\mathrm{H} 24) .{ }^{10}$ Chart $1 \mathrm{~A}$ shows the chemical structure of BMVC. Significant increase of fluorescence yield and distinctive fluorescence properties of the BMVC upon binding to various DNA structures could allow us to verify the presence of G-quadruplex structures in human telomeres. ${ }^{8}$ However, the localization of the G-quadruplex structures in metaphase chromosomes was not directly visualized. Moreover, two types of G-quadruplex structures of the human telomeres have been documented in vitro. ${ }^{11-14}$ Structures B and C in Chart I show two different G-quadruplex structures of $\mathrm{d}\left[\mathrm{AG}_{3}\left(\mathrm{~T}_{2} \mathrm{AG}_{3}\right)_{3}\right]$ ( $\left.\mathrm{H} 22\right)$

(3) Mergny, J. L.; Hélène, C. Nat. Med. 1998, 4, 1366-1367.

(4) Kerwin, S. M. Curr. Pharm. Des. 2000, 6, 441-471.

(5) Han, H.; Hurley, L. H. Trends Pharmacol. Sci. 2001, 21, 136-142.

(6) Schaffitzel, C.; Berger, I.; Postberg, J.; Hanes, J.; Lipps, H. J.; Plückthun, A Proc. Natl. Acad. Sci. U.S.A. 2001, 98, 8572-8577.

(7) Siddiqui-Jain, A.; Grand, C. L.; Bearss, D. J.; Hurley, L. H. Proc. Natl. Acad. Sci. U.S.A. 2002, 99, 11593-11598.

(8) Chang, C. C.; Kuo, I.-C.; Ling, I.-F.; Chen, C. T.; Chen, H. C.; Lou, P. J.; Lin, J. J.; Chang, T.-C. Anal. Chem. 2004, 76, 4490-4494.

(9) Granotier, C.; Pennarun, G.; Riou, L.; Hoffschir, F.; Gauthier, L. R.; Cian, A. D.; Gomez, D.; Mandine, E.; Riou, J.-F.; Mergny, J.-L.; Mailliet, P.; Dutrillaux, B.; Boussin, F. D. Nucleic Acids Res. 2005, 33, 4182-4190.

(10) Chang, C. C.; Wu, J. Y.; Chien, C. W.; Wu, W. S.; Liu, H.; Kang, C. C.; Yu, L. J.; Chang, T.-C. Anal. Chem. 2003, 75, 6177-6183.

(11) Wang, Y.; Patel, D. J. Structure 1993, 1, 262-283.

(12) Neidle, S.; Parkinson, G. N. Curr. Opin. Struct. Biol. 2003, 13, 275-283. 


\section{Chart 1}

(A)

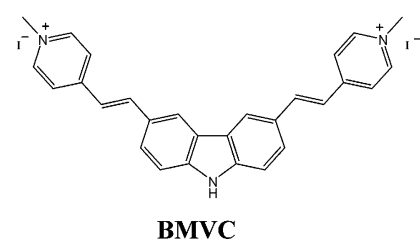

(B)

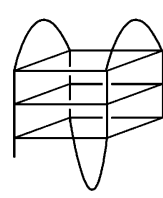

NMR (Na)

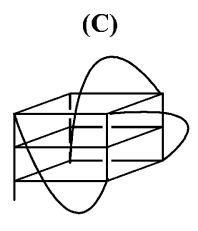

X-ray (K) determined by NMR in the presence of sodium ${ }^{11}$ and X-ray diffraction in the presence of potassium, ${ }^{12}$ respectively. To the best of our knowledge, direct identification of a specific G-quadruplex structure in native human telomeres has not been reported. Verification of the G-quadruplex structures in vivo is crucial in the design of structure-specific DNA binding ligands, especially for the development of anticancer agents. ${ }^{3-5}$

Similar fluorescence spectra of BMVC upon interaction with $\mathrm{H} 24$ in the presence of $\mathrm{Na}^{+}$and $\mathrm{K}^{+}$solutions cannot be used to distinguish their different G-quadruplex structures. Since the lifetime of a fluorophore could be different due to the influence of the environment, ${ }^{15}$ it is worth measuring the fluorescence lifetimes of BMVC upon interaction with different DNAs. In addition, the powerful technique of two-photon excitation (2PE) fluorescence lifetime imaging microscopy (FLIM) has been recently applied to monitor the dye-DNA interaction and map protein localization in living cells. ${ }^{16-18}$ Here we have applied 2PEFLIM to measure the fluorescence lifetime of BMVC in the presence of $\mathrm{d}\left(\mathrm{T}_{2} \mathrm{AG}_{3}\right)_{4}$ in sodium solution to be $1.95 \pm 0.25 \mathrm{~ns}$. This lifetime differs from the $1.5 \pm 0.2 \mathrm{~ns}$ upon interaction with two DNA duplexes and the $1.56 \pm 0.18 \mathrm{~ns}$ in the presence of $\mathrm{d}\left(\mathrm{T}_{2} \mathrm{AG}_{3}\right)_{4}$ in potassium solution. The distinct long-lifetime component allows us to use a discrete time mode in 2PE-FLIM image for the first time to verify the presence of the antiparallel G-quadruplex structure, as shown in Chart 1B, in human telomeres and to map its localization in metaphase chromosomes of nasopharyngeal carcinoma $\mathrm{KJ}-1$ cells.

\section{EXPERIMENTAL SECTION}

DNA Samples. All DNA oligonucleotides were purchased from Applied Biosystems. A buffer solution ( $\mathrm{pH}$ 7.5) that consisted of $10 \mathrm{mM}$ Tris- $\mathrm{HCl}$ and $150 \mathrm{mM} \mathrm{NaCl}$ (or $10 \mathrm{mM} \mathrm{NaCl} / 140 \mathrm{mM}$ $\mathrm{KCl}$ ) was used for all experiments. Buffer solutions mixed with DNA oligonucleotides were heated to $90{ }^{\circ} \mathrm{C}$ for $2 \mathrm{~min}$, cooled slowly to room temperature, and then stored for more than 2 days at $4{ }^{\circ} \mathrm{C}$ before use.

BMVC Labeling of Metaphase Chromosomes. To obtain cells arrested at metaphase of the cell cycle, cells were first cultivated in DMEM containing $10 \% \mathrm{FCS}$ at $37{ }^{\circ} \mathrm{C}$ in a $5 \% \mathrm{CO}_{2}$ incubator. Subconfluent monolayer cells were subjected to $1 \mu \mathrm{g} /$

(13) Rujan, I. N.; Meleney, J. C.; Bolton, P. H. Nucleic Acids Res. 2005, 33, 20222031.

(14) Li, J.; Correia, J. J.; Wang, L.; Trent, J. O.; Chaires, J. B. Nucleic Acids Res. 2005, 33, 4649-4659.

(15) Edman, L.; Mets, U.; Rigler, R. Proc. Natl. Acad. Sci. U.S.A. 1996, 93, 67106715.

(16) Lakowicz, J. R.; Gryczynski, I.; Maslak, H.; Schrader, M.; Engelhardt, P.; Kano, H.; Hell, S. W. Biophys. J. 1997, 72, 567-578.

(17) Chen, Y.; Periasamy, A. Microsc. Res. Technol. 2004, 63, 72-80.

(18) Errington, R. J.; Ameer-beg, S. M.; Vojnovic, B.; Patterson, L. H.; Zloh, M.; Smith, P. J. Adv. Drug Delivery Rev. 2005, 57, 153-167.
mL Demecolcine (Colcemid, Life Technologies, Gaithersburg, $\mathrm{MD})$ treatment for $1 \mathrm{~h}$. The arrested cells were resuspended in $0.075 \mathrm{M} \mathrm{KCl}$ hypotonic buffer for $20 \mathrm{~min}$ and then fixed with methanol/acetic acid (3:1). An aliquot of $15-20 \mu \mathrm{L}$ of cell suspension was dropped on to a slightly humidified slide and then the slide placed on a hot metal plate to evaporate the fixatives. The sample was washed with PBS and then incubated with 0.1 $\mu \mathrm{M}$ BMVC for $90 \mathrm{~s}$ at room temperature. After incubation, the sample was washed again before imaging study.

Absorption, Fluorescence, and Circular Dichroism. Absorption spectra were taken on a Hitachi U3200 UV-visible spectrophotometer, and fluorescence spectra were recorded on a Hitachi F4010 spectrofluorometer with a $2 \mathrm{~nm}$ band-pass in a 1 $\mathrm{cm}$ cell length at room temperature. The circular dichroism spectra averaged 10 scans on a Jasco J-715 spectropolarimeter with $2 \mathrm{~nm}$ bandwidth at a scan speed of $50 \mathrm{~nm} / \mathrm{min}$ and a step resolution of $0.2 \mathrm{~nm}$. The circular dichroism spectra were measured under $\mathrm{N}_{2}$ over the range of $210-350 \mathrm{~nm}$ to monitor the G-quadruplex structures.

Competition Binding Assay. To perform the competition binding assay, $0.5 \mu \mathrm{M}$ BMVC was incubated with $20 \mu \mathrm{M}$ linear duplex [d(ATGCGCA T $_{2}$ GCGCAT) $]_{2}$ (D16 duplex), a G-rich quadruplex H24, and a telomeric duplex d $\left(\mathrm{T}_{2} \mathrm{AG}_{3}\right)_{4} / \mathrm{d}\left(\mathrm{C}_{3} \mathrm{TA}_{2}\right)_{4}(\mathrm{Tel}$ duplex) and their mixtures of (D16 + H24), (D16 + Tel), and $(\mathrm{H} 24+\mathrm{Tel})$ for $10 \mathrm{~min}$ in buffer containing $10 \mathrm{mM}$ Tris-HCl and $140 \mathrm{mM} \mathrm{KCl}(\mathrm{pH} 7.5)$. The reaction products were analyzed using $20 \%$ polyacrylamide gels. The gels were run at $100 \mathrm{~V} / \mathrm{cm}$ for $15 \mathrm{~h}$ at $4{ }^{\circ} \mathrm{C}$. To confirm the presence of quadruplex and linear duplex DNA, the same gels were then poststained in a solution containing $10 \mu \mathrm{M}$ of BMVC for $10 \mathrm{~s}$.

2PE-FLIM Method. The 2PE-FLIM experiment was conducted by using a femtosecond laser pulse at $810 \mathrm{~nm}$ generated by a mode-locked Ti:sapphire laser (Mira-900, Coherent) with $\sim 150$ fs pulse width at $76 \mathrm{MHz}$ repetition rate. The irradiation laser power after the objective $(60 \times, \mathrm{NA}=1.4$, Olympus $)$ is $\sim 5$ $\mathrm{mW}$ throughout the measurements. The raster scanning mapping mechanism is achieved by a modified laser-scanning microscope (FV300, Olympus). The fluorescence of BMVC followed a quadratic dependence of the excitation intensity. Synchronized timecorrelated single-photon counting (TCSPC) module (SPC830, Becker \& Hickle) was used to collect the fluorescence signal that is used to construct the 2PE-FLIM images. The lifetime analysis on 2PE-FLIM micrograph could be measured on pixel-by-pixel basis in discrete areas.

\section{RESULTS AND DISCUSSION}

Circular Dichroism Spectra of $\mathbf{d}\left(\mathrm{T}_{2} \mathrm{AG}_{3}\right)_{4}$. Figure $1 \mathrm{~A}$ shows circular dichroism spectra of H24 DNA in the presence of 150 $\mathrm{mM}$ sodium (H24-Na) or $10 \mathrm{mM}$ sodium/140 mM potassium (H24-K). In addition, the spectra show no appreciable difference upon interaction with BMVC. Different circular dichroism patterns of $\mathrm{H} 24$ in $\mathrm{Na}^{+}$and $\mathrm{K}^{+}$solutions suggest the existence of different G-quadruplex structures. Our circular dichroism patterns are very similar to the patterns reported by Bolton and co-workers. ${ }^{13}$ Wang and Pate ${ }^{11}$ identified the NMR structure in solution characterized by an antiparallel G-quadruplex structure with two lateral loops at one end of the G-quartet and a diagonal loop at the other end of the quartet in the presence of sodium, as shown in Chart 1B. Neidle and Parkinson ${ }^{12}$ determined the crystal structure of a 


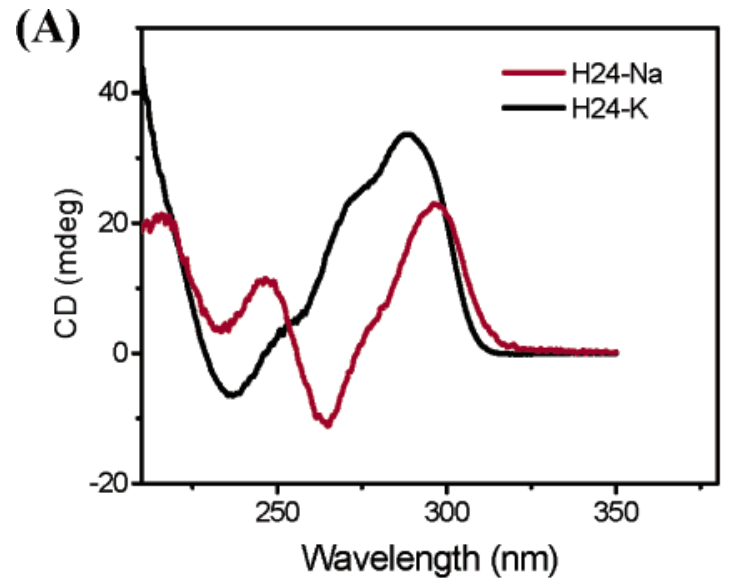

(B)

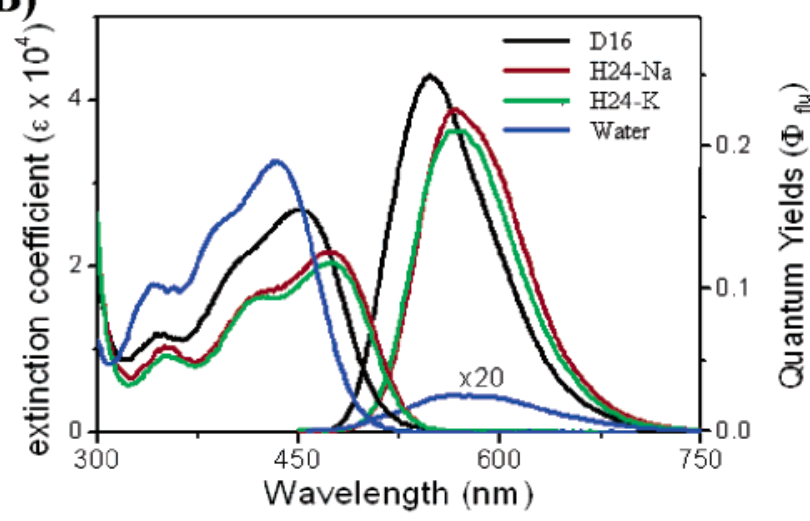

(C)

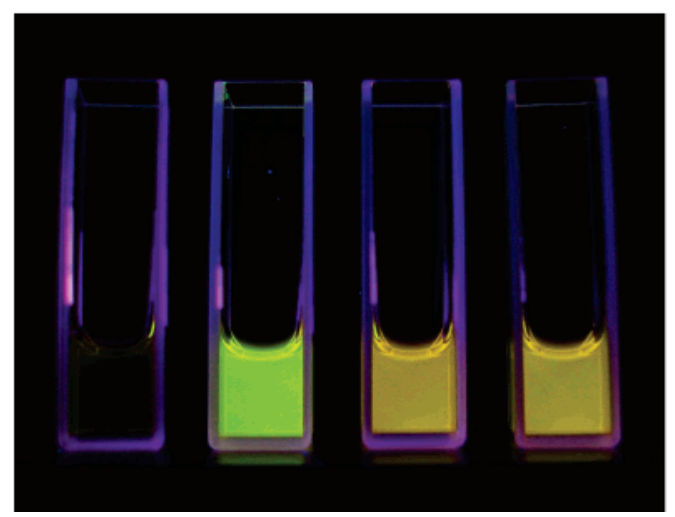

Figure 1. (A) Circular dichroism spectra of $d\left(T_{2} A G_{3}\right)_{4}(H 24)$ in the presence of $150 \mathrm{mM}$ sodium $(\mathrm{H} 24-\mathrm{Na})$ and $10 \mathrm{mM}$ sodium/140 mM potassium (H24-K). (B) Absorption and fluorescence spectra of BMVC and its complexes with a linear duplex [d(ATGCGCA $\mathrm{T}_{2}$ GCGCAT) $]_{2}$ (D16 duplex), H24-Na and H24-K quadruplexes. (C) Fluorescence of BMVC and its complexes with D16, H24-Na, and H24-K (from left to right) under UV illumination and recorded by a conventional color camera.

parallel G-quadruplex structure with three external loops located in the grooves by X-ray diffraction in the presence of potassium, as shown in Chart $1 \mathrm{C}$. This type of parallel G-quadruplex structure was also found in the human c-myc promoter by the NMR study. ${ }^{19}$ Bolton et al. ${ }^{13}$ suggested that the parallel G-quadruplex structure occurs in the presence of $10 \mathrm{mM}$ sodium and $140 \mathrm{mM}$ potassium, while the antiparallel G-quadruplex structure appears in the

(19) Phan, A. T.; Modi, Y. S.; Patel, D. J. J. Am. Chem. Soc. 2004, 126, 87108716. presence of $150 \mathrm{mM}$ sodium. In contrast, Chaires et al. ${ }^{14}$ suggested that the structure of the $\mathrm{H} 24$ in $\mathrm{K}^{+}$solution is not the parallel G-quadruplex structure as determined in the crystalline structure. Although different G-quadruplex structures of $\mathrm{H} 24$ in $\mathrm{K}^{+}$solution were proposed, ${ }^{13,14}$ the antiparallel G-quadruplex structure of H24 in $\mathrm{Na}^{+}$solution, as shown in Chart $1 \mathrm{~B}$, has been widely accepted.

Absorption and Fluorescence of BMVC and Its Complexes with Various DNAs. Figure $1 \mathrm{~B}$ shows the absorption and fluorescence spectra of $20 \mu \mathrm{M}$ BMVC and its complexes with various DNA structures of H24-Na and H24-K quadruplexes and D16 duplex. Figure 1C demonstrates their corresponding fluorescence of BMVC under illumination with a UV lamp and recorded by a conventional color camera. Significant increase of fluorescence emissions of BMVC upon interaction with DNA together with distinct fluorescence emissions of BMVC at $~ 575$ $\mathrm{nm}$ in $\mathrm{H} 24$ and $~ 545 \mathrm{~nm}$ in D16 DNA allows us to distinguish the quadruplex structures from the duplex structures. However, very similar fluorescence emissions of BMVC in $\mathrm{H} 24-\mathrm{Na}$ and H24-K cannot be used to distinguish different G-quadruplex structures in human telomeres.

The marked increase of the fluorescence quantum yield of certain fluorophores upon binding to DNA has been documented elsewhere. For example, auramine shows weak fluorescence in water but strong fluorescence in DNA. ${ }^{20}$ The very low quantum yield of auramine in water was attributed to intramolecular charge transfer in the excited state of auramine. ${ }^{21}$ Castex et al. ${ }^{22}$ have documented that an intramolecular charge-transfer state can be formed in carbazole derivatives when the electron-acceptor groups are linked to the electron-donor carbazole molecule. Very recently, Diau et al. ${ }^{23}$ have studied relaxation dynamics of 3,6-distyrylcarbazoles and found that the localization of the $\pi$-conjugation and the bridged $\mathrm{CC}$ bond twisting motion on the excited state are responsible for the weak fluorescence quantum yield in solution. Therefore, we suggest that the environmentally sensitive fluorescence of BMVC can be rationalized on the basis of the intramolecular charge-transfer processes between the electron donor of carbazole moiety and the electron acceptor of methylpyridinium cation perturbed by the rotation motion of the vinyl group.

Competition Binding Assays in the Presence of Potassium. In our previous work, ${ }^{10}$ the competition binding assay showed that the BMVC has better binding preference to H24 quadruplex than D16 duplex in the presence of sodium. To evaluate the possible components of different G-quadruplex structures of $\mathrm{H} 24$ in potassium solution and determine the binding preference of BMVC to various DNA, Figure 2A shows the prestained gels of $0.5 \mu \mathrm{M}$ BMVC incubated with D16 duplex, H24 quadruplex, and Tel duplex in the presence of potassium. As a control, the same gels were then poststained with $10 \mu \mathrm{M}$ BMVC to visualize the position and level of DNA loaded, as shown in Figure 2B. Under our electrophoresis condition, BMVC-bound H24 complex migrates faster than that of free H24. Thus, both BMVC-bound and free quadruplex H24 could be revealed in the poststained gels. It is found that one major component of $\mathrm{H} 24-\mathrm{K}$

(20) Oster, G.; Nishijima, Y.J. Am. Chem. Soc. 1956, 78, 1581-1584.

(21) Changenet, P.; Zhang, H.; van der Meer, M. J.; Glasbeck, M.; Plaza, P.; Martin, M. M. J. Phys. Chem. A 1998, 102, 6716-6721.

(22) Castex, M. C.; Olivero, C.; Pichler, G.; Adés, D.; Cloutet, E.; Siove, A. Synth. Met. 2001, 122, 59-61.

(23) Wang, T. S.; Chung, S. M.; Wu, F. I.; Shu, C. F.; Diau, W. G. J. Phys. Chem. B. 2005, 109, 23827-23835. 


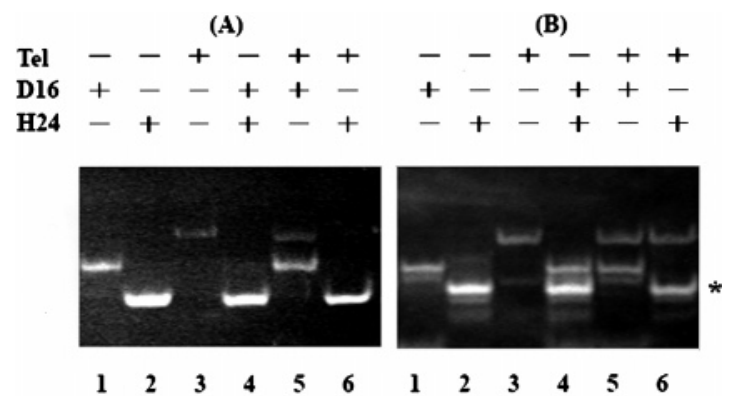

Figure 2. Binding preference of BMVC to the G-quadruplex structure of $\mathrm{H} 24$ in the presence of potassium. (A) Prestained gels of each $20 \mu \mathrm{M} \mathrm{D} 16$ (1), H24 (2), and Tel (3), and the mixtures of D16 and $\mathrm{H} 24$ (4), D16 and Tel (5), and $\mathrm{H} 24$ and Tel (6) incubated with $0.5 \mu \mathrm{M}$ BMVC and analyzed on a $20 \%$ polyacrylamide gels. (B) The same gels were then poststained with $10 \mu \mathrm{M} \mathrm{BMVC}$ to visualize the position and level of DNA loaded. An asterisk indicates position of the free quadruplex DNA.

is detected in the presence of potassium (Figure 2B, lane 2). Since there is also one major component of $\mathrm{H} 24-\mathrm{Na}$ in the presence of sodium, ${ }^{10}$ different circular dichroism patterns of H24-Na and H24-K reveal their different G-quadruplex structures. In the prestained competition gels, BMVC fluorescence was only detected in H24 quadruplex when a limiting amount of BMVC was used (Figure 2A, lanes 4,6). It appears that BMVC has better binding preference toward H24 quadruplex than D16 and Tel duplexes. Similar results were also found in the presence of sodium. In addition, the competition analysis shows that the binding affinity of BMVC to D16 duplex is higher than Tel duplex (Figure 2A, lane 5). Nevertheless, BMVC has similar a binding preference to the G-quadruplex structures of $\mathrm{H} 24-\mathrm{Na}$ and $\mathrm{H} 24-\mathrm{K}$ over duplexes.

Fluorescence Decay Times of BMVC upon Interaction with Different DNAs. We now apply 2PE-FLIM to measure the fluorescence lifetimes of BMVC upon interaction with various DNAs. Figure 3A shows typical decay curves of BMVC in the presence of D16 duplex and H24-Na quadruplex fitted by singleexponential lifetimes of 1.46 and $1.99 \mathrm{~ns}$, respectively. The sample was spin-coated on a coverslip, and the decay time was measured by using a TCSPC equipped with a femtosecond laser pulse at $810 \mathrm{~nm}$. Figure 3B shows the histograms of the fluorescence lifetimes of BMVC upon interaction with $\mathrm{H} 24-\mathrm{Na}$ and $\mathrm{H} 24-\mathrm{K}$ quadruplexes and D16 duplex characterized by $1.96 \pm 0.22,1.56$ \pm 0.18 , and $1.46 \pm 0.15 \mathrm{~ns}$, respectively. In addition, the decay time of BMVC upon interaction with Tel duplex was measured to be $1.53 \pm 0.13 \mathrm{~ns}$. Although we are not able to distinguish the $\mathrm{H} 24-\mathrm{K}$ quadruplex from the duplexes at present, the long lifetime of BMVC upon interaction with $\mathrm{H} 24-\mathrm{Na}$ can be applied to determine whether the antiparallel G-quadruplex structure exists in native human telomeres. Moreover, different fluorescence lifetimes of BMVC together with different circular dichroism spectra support that H24-Na and H24-K have different G-quadruplex structures. Considering the gel assay, distinct fluorescence lifetimes of BMVC measured in H24-Na and H24-K indicate that the hypothesis of the coexistence of the two G-quadruplex structures in $\mathrm{H} 24-\mathrm{K}$ is unlikely. ${ }^{24}$

(24) Ying, L.; Green, J. J.; Li, H.; Klenerman, D.; Balasubramanian, S. Proc. Natl. Acad. Sci. U.S.A. 2003, 100, 14629-14634.
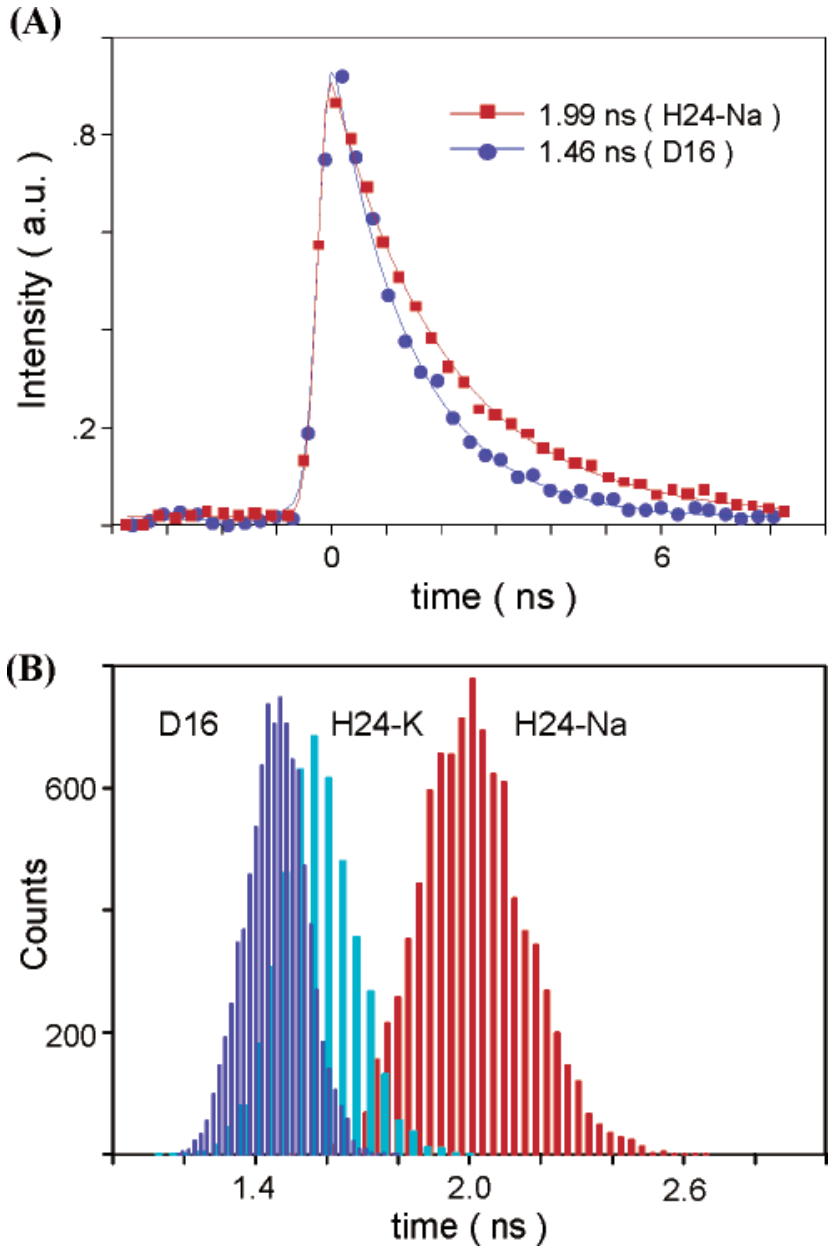

Figure 3. Analysis on TCSPC-based 2PE-FLIM micrograph of 10 $\mu \mathrm{M}$ DNA incubated with $0.1 \mu \mathrm{M}$ BMVC for $10 \mathrm{~min}$. (A) Fluorescence decay time of BMVC in the presence of D16 and H24-Na fitted by single-exponential decay curve. (B) Histograms of BMVC fluorescence decay times in the presence of D16, H24-Na, and H24-K.

Localization of the Antiparallel Quadruplex Structure in Metaphase Chromosomes by Using 2PE-FLIM. Considering the overwhelmed duplex structures of DNA in chromosomes, we take the advantages of the binding preference and high sensitivity of BMVC toward G-quadruplex structures of H24 over duplexes to verify whether the antiparallel G-quadruplex structures exist in human telomeres. The metaphase chromosomes prepared from nasopharyngeal carcinoma $\mathrm{KJ}-1$ cells on microscope slides were incubated with a low concentration $0.1 \mu \mathrm{M}$ BMVC for a short time $90 \mathrm{~s}$ before the imaging study. Figure $4 \mathrm{~A}$ shows a typical $2 \mathrm{PE}-$ FLIM micrograph of metaphase chromosomes of $\mathrm{KJ}-1$ cells stained by BMVC. Figure $4 \mathrm{~B}$ shows the corresponding distribution of the fluorescence decay time of BMVC. Considering the huge excess in duplex DNA in chromosomes, it is reasonable that a very tiny amount of long-lifetime component is present in Figure 4B. We have further used the discrete time mode at $1.85 \mathrm{~ns}$ to identify the localization of the long-lifetime component for visualizing the antiparallel G-quadruplex structure in metaphase chromosomes, as shown in Figure 4C. For clarity, red is designated as mode 1 resulting from the interaction with antiparallel G-quadruplex structure characterized by the lifetime in the region of $1.85-2.2$ ns, while white is designated as mode 2 reflecting the interaction with other DNA structures characterized by the lifetime in the 
(A)

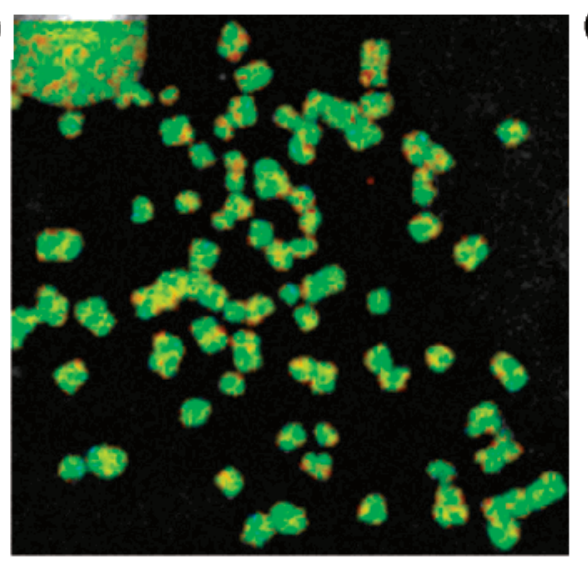

(B)

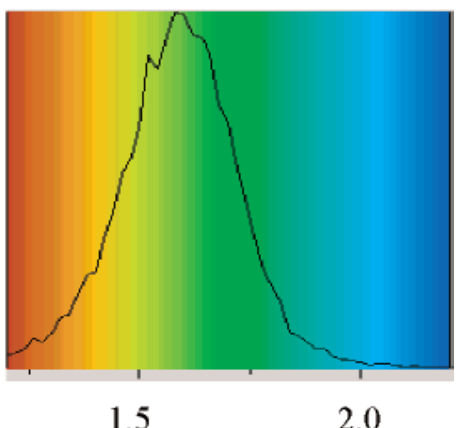

1.5 Lifetime (ns)

(C)

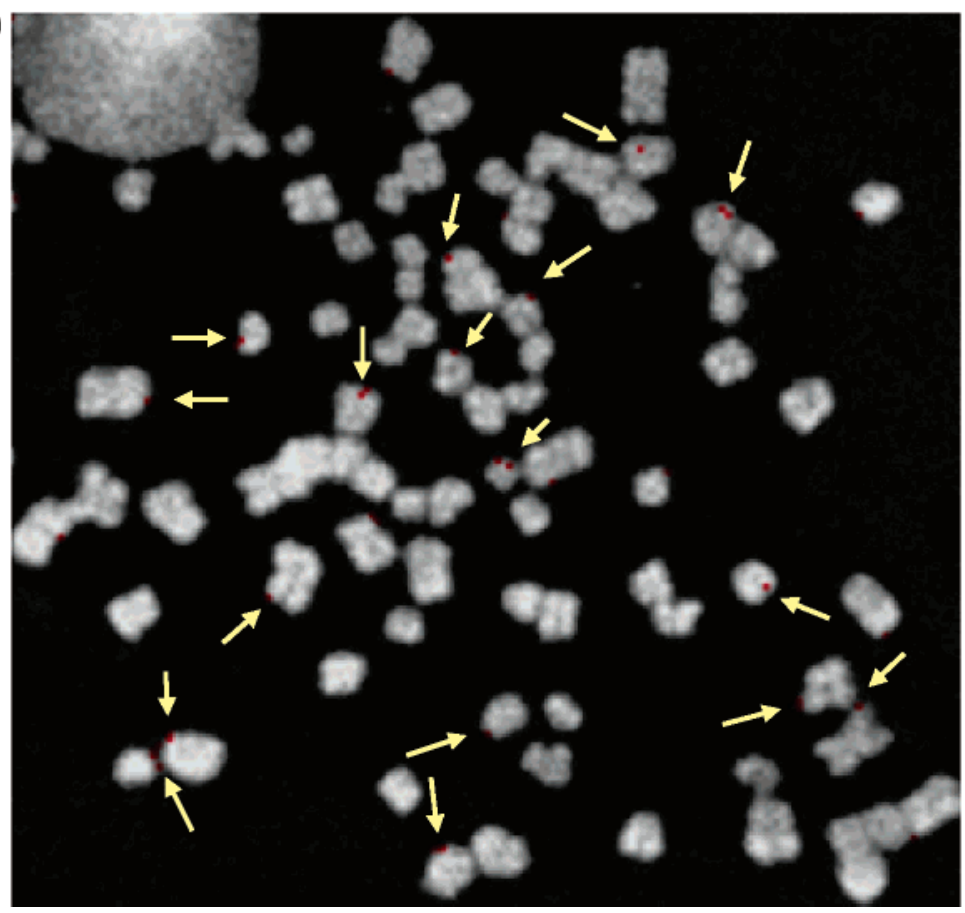

Figure 4. $2 \mathrm{PE}$-FLIM micrograph of BMVC-stained metaphase chromosomes of nasopharyngeal carcinoma KJ-1 cells. (A) A typical $2 \mathrm{PE}-\mathrm{FLIM}$ image of metaphase chromosomes of nasopharyngeal carcinoma KJ-1 cells stained with $0.1 \mu \mathrm{M}$ BMVC for $90 \mathrm{~s}$. (B) The fluorescence lifetime distribution of the BMVC-stained chromosomes. (C) The color-coded image of the metaphase chromosomes is represented by discrete time mode at $1.85 \mathrm{~ns}$. Red designates mode 1 resulting from the interaction with antiparallel G-quadruplex structures, while white designates mode 2 due to the interaction with other DNA structures. The arrows indicate mode 1 for visualization.

region of $1.2-1.85 \mathrm{~ns}$. Although mode 2 dominates in most of the areas of the chromosomes, the key finding is that mode 1 appears mostly in the ends of the chromosomes. Here the 2PEFLIM data provide the first clear evidence of the presence of antiparallel G-quadruplex structure in native human telomeres.

Further statistical measurements of individual chromosomes stained by $0.1 \mu \mathrm{M}$ BMVC show that mode 1 appears in human telomeres of KJ-1 cells to be $46.69 \%(127 / 272)$ by using the discrete time mode at $1.85 \mathrm{~ns}$. Note that the ratio between mode 1 and mode 2 (mode 1/mode 2) of individual chromosomes decreases as the staining BMVC concentration increases. This is attributed to the binding preference to H24 quadruplex over D16 duplex that allows the BMVC to bind to the H24 quadruplex first even in the presence of excess amount of duplex DNA. It should be noted that the detection of the antiparallel G-quadruplex structure characterized by a $1.96 \mathrm{~ns}$ lifetime component from cells does not rule out the parallel G-quadruplex structure characterized by a $1.56 \mathrm{~ns}$ lifetime component. Considering that there is more potassium than sodium in cells, it is likely that the H24-K quadruplex could exist in human telomeres. However, the quadruplex structure of H24 in potassium solution is currently in debate. ${ }^{9,13,14}$ Determination of the H24-K structure, verification of the H24-K structure in human telomeres, and measurement of the relative ratio between $\mathrm{H} 24-\mathrm{K}$ and $\mathrm{H} 24-\mathrm{Na}$ in human chromosomes deserve more detailed studies.

In addition, it is of interest to find some distinct mode 1 spots along the edge of the chromosomes. Particularly, the mode 1 spots occur at the center of chromosomes with a total statistical measurement of 3.31\% (9/272). It implies that the 2PE-FLIM data of BMVC can be applied to map the distribution and localization of the antiparallel G-quadruplex structure in chromosomes. Considering the appearance of G-quadruplex structures in other regions, such as human insulin ${ }^{25}$ and the upstream of the P1 promoter in the c-myc of the human genes, ${ }^{7,19,26}$ the localization 
of the antiparallel G-quadruplex structure in chromosomes presents an interesting subject.

The existence of G-quadruplex structures in human telomeres and the distribution of G-quadruplex structures in chromosomes constitute the central issues in establishing the importance of quadruplex stabilizers in antitumor agents and telomere biology in cancer research. The key finding is that the localization of antiparallel G-quadruplex structure is detected not only in native human telomeres but also in other regions of the chromosomes. In addition, the TCSPC-based 2PE-FLIM method on a novel BMVC probe opens a new way to study dye-DNA interactions as well as understanding fundamental aspects of telomere biology

(25) Catasti, P.; Chen, X.; Moyzis, R. K.; Bradbury, E. M.; Gupta, G. J. Mol. Biol. 1996, 264, 534-545.

(26) Seenisamy, J.; Rezler, E. M.; Powell, T. J.; Tye, D.; Gokhale, V.; Joshi, C. S.; Siddiqui-Jain, A.; Hurley, L. H. J. Am. Chem. Soc. 2004, 126, 8702-8709. in cells, such as monitoring the antiparallel G-quadruplex structure in telomeres or in chromosomes after anticancer drug treatment. In addition, it would be of great interest to determine whether the existence of antiparallel G-quadruplex structure in human telomeres depends on the specific types of chromosomes.

\section{ACKNOWLEDGMENT}

This work was supported by Academia Sinica, the Imaging Core at Yang-Ming University, and the National Science Council of the Republic of China (Grants NSC-93-2113-M001-044 and NSC93-2112-M-110-002).

Received for review December 15, 2005. Accepted February 1, 2006.

AC052218F 